\title{
Level Crossing Analysis of BER for Adaptive MIMO OFDM Systems
}

\author{
Krishna P. Kongara Peter J. Smith \\ Department of Electrical and Computer Engineering, \\ University of Canterbury, Christchurch, New Zealand
}

\begin{abstract}
In this paper we consider the level crossing rates (LCRs) of bit error rates (BERs) across the frequency bins of a multiple-input multiple-output (MIMO) system with orthogonal frequency division multiplexing (OFDM) operating over frequency selective Rayleigh fading environments. In particular, we consider each eigenmode as a random process in the frequency domain and compute the LCR for the BER of transmission down the eigenchannels of the MIMO OFDM channel. Many extensions to this work are possible including LCRs in time as well as in frequency. The accuracy of the analytical approximations are verified by Monte Carlo simulations.
\end{abstract}

\section{INTRODUCTION}

Multiple antennas at the transmitter and receiver create a MIMO wireless channel and can be used to provide spatial diversity to combat fluctuations in signal strength or fading in wireless channels $[1,2,3,4]$. MIMO wireless systems have been shown to provide a diversity gain equal to the product of the number of transmit antennas and the number of receive antennas in narrowband channels. For the case of complete knowledge of the channel state information (CSI) at the transmitter, full diversity can be achieved using transmit beamforming and receive combining $[5,6,7]$. Compared to space-time block codes, beamforming and combining provide additional array gain and a lowcomplexity and flexible receiver structure. The CSI at the transmitter may be obtained from the receiver using a feedback control channel. The particular case of beamforming and combining using a feedback channel to convey the CSI has been investigated in $[8,9]$.

LCR is an important second order statistic to gauge the time-varying, frequency selective nature of the channel and system performance. LCR is defined as the average number of times that a signal down-crosses (or up-crosses) a certain threshold per unit time. LCR is useful in many aspects of receiver design such as dynamic range, equalization, diversity, modulation schemes, and error control coding $[10,11,12,13,14]$.

In [15], we have provided novel results (in the frequency domain) on the LCR of the maximum eigenvalue of a MIMO OFDM system. In this paper we extend our analysis to calculating the LCR of BER in an adaptive MIMO OFDM system which may use multiple eigenchannels. Our results provide new insights into the BER analysis of an adaptive system. It is worth reiterating that we focus on BER as a process in frequency across the OFDM block. Hence we consider variations in BER across the bins and not over time.

The rest of this paper is organized as follows. In Sec. 2 we outline the key assumptions and notation. In addition, we present some fundamental background and mathematical formulations for MIMO OFDM systems and adaptive modulation. The LCR analysis is presented in Sec. 3. We verify our analysis using Monte Carlo simulations in Sec. 4, and conclusions are given in Sec. 5.

\section{MIMO OFDM SYSTEM}

For convenience we give a system description in this section for the case where only the maximum eigenchannel is used. However, as shown in Sec. 3.1, the analysis is simple to extend to multiple eigenchannels. Hence, we consider an adaptive MIMO OFDM beamforming system transmitting over $N$ subcarriers with $N_{T}$ antennas at the transmitter and $N_{R}$ antennas at the receiver. The system transmits data symbol $S_{k}$ on the $k$-th subcarrier for $k \in\{1,2, \ldots, N\}$, where $S_{k} \in \mathbb{R}^{2}$ is from some two-dimensional symbol constellation. We refer to the superposition of all $N$ modulated subcarriers as the OFDM block. We assume that each subcarrier occupies a subchannel of bandwidth $\Delta f(\mathrm{~Hz})$, yielding a total bandwidth of $B=N \Delta f$. Furthermore, each subcarrier symbol is transmitted with equal en- 
ergy $E_{s}$ such that the total average transmitted energy is $E_{N}=N E_{s}$.

At the transmitter, the $k$-th subcarrier modulates the symbol $S_{k}$ using the beamforming vector (or weight vector) $\boldsymbol{b}_{k}$. We assume that the sampled impulse response of the channel is shorter than the cyclic prefix. After removing the cyclic prefix, the channel for the $k$-th subcarrier after the Discrete Fourier Transform (DFT) can then be described as a $N_{R} \times N_{T}$ complex channel matrix $\boldsymbol{H}_{k}$. Considering a beamformingcombining system, the output of the combiner at the receiver on the $k$-th subcarrier can be written as

$$
R_{k}=\boldsymbol{z}_{k}^{\dagger} \boldsymbol{H}_{k} \boldsymbol{b}_{k} S_{k}+\boldsymbol{z}_{k}^{\dagger} \boldsymbol{n}_{k}
$$

where $\dagger$ represents the conjugate transpose, $\boldsymbol{z}_{k}$ is the combiner weight vector and $\boldsymbol{H}_{k}$ is the narrowband channel transfer function for subcarrier $k$. The noise vector is denoted by $\boldsymbol{n}_{k}$ with independent and identically distributed (i.i.d.) Gaussian entries distributed according to $\mathcal{C} \mathcal{N}\left(0, \sigma^{2}\right)$. We set $\left\|\boldsymbol{b}_{k}\right\|=1$ to reflect the power constraint at the transmitter, where $\|\cdot\|$ denotes the Euclidian norm.

For a given beamforming vector $\boldsymbol{b}_{k}$, the combining vector $\boldsymbol{z}_{k}$ that maximizes the SNR is given by [8]

$$
\boldsymbol{z}_{k}=\frac{\boldsymbol{H}_{k} \boldsymbol{b}_{k}}{\left\|\boldsymbol{H}_{k} \boldsymbol{b}_{k}\right\|}
$$

Then, varying the beamforming vector, the maximum SNR is achieved if $\boldsymbol{b}_{k}$ is proportional to the eigenvector corresponding to the maximum eigenvalue $\lambda_{\max }^{(k)}$ of $\boldsymbol{H}_{k} \boldsymbol{H}_{k}^{\dagger}$. This transmission scheme is commonly described as maximum ratio transmission and maximum ratio combining (MRT-MRC), which achieves full diversity and full array gain in Rayleigh fading channels $[5,8]$. Substituting the eigenvector solution for $\boldsymbol{b}_{k}$ into (2), the resulting optimal SNR can be written as

$$
\gamma_{\max }^{(k)}=\frac{E_{s}}{\sigma^{2}} \lambda_{\max }^{(k)}
$$

where $\frac{E_{s}}{\sigma^{2}}$ denotes the average SNR per branch, and (1) can be replaced by

$$
R_{k}=\sqrt{\lambda_{\max }^{(k)}} S_{k}+\widetilde{n}
$$

where $\tilde{n} \sim \mathcal{C N}\left(0, \sigma^{2}\right)$ is a complex Gaussian noise term independent of $\lambda_{\max }^{(k)}$. From (3) we see that the subcarrier SNR, $\gamma_{\max }^{(k)}$ is proportional to $\lambda_{\max }^{(k)}$. Hence, the $\mathrm{BER}$ is a function of the maximum eigenvalue. This simple formulation of the received signal is a necessary result for the forthcoming LCR analysis.

\subsection{Frequency Selective Channel}

We assume a familiarity with frequency selective Rayleigh fading channels and use the well-known Jakes' model [16]. We make the general assumption of a frequency selective Rayleigh fading channel that is wide sense stationary with uncorrelated, isotropic scattering. Furthermore, we presume that the delay autocorrelation function may be described as an exponential delay power profile with rms delay $\tau_{d}$. However, note that the analysis developed later does not depend on the type of delay power profile. We select an arbitrary time point and only consider variation across frequency. Consider the $(i, j)^{t h}$ elements of $\boldsymbol{H}_{k 1}$ and $\boldsymbol{H}_{k_{2}}$, denoted by $H_{k_{1}}$ and $H_{k_{2}}$ respectively, for $i=1,2, \ldots . ., N_{R}$, $j=1,2, \ldots ., N_{T}$. These gains can be written as

$$
H_{k_{1}}=X_{k_{1}}+j Y_{k_{1}} \quad \text { and } \quad H_{k_{2}}=X_{k_{2}}+j Y_{k_{2}}
$$

where $X_{k_{1}}, Y_{k_{1}}, X_{k_{2}}$ and $Y_{k_{2}}$ are identically distributed zero mean Gaussian random variables. Without loss of generality we may set $\mathrm{E}\left[X_{k}^{2}\right]=\mathrm{E}\left[Y_{k}^{2}\right]=\frac{1}{2}$, for all $k$. Following [16], we may then write the cross-correlations

$$
\begin{aligned}
\mathrm{E}\left[X_{k_{1}} X_{k_{2}}\right] & =\mathrm{E}\left[Y_{k_{1}} Y_{k_{2}}\right]=\frac{1}{2} \frac{1}{1+\left(2 \pi \tau_{d} \Delta f \Delta k\right)^{2}}, \\
\mathrm{E}\left[X_{k_{1}} Y_{k_{1}}\right] & =\mathrm{E}\left[X_{k_{2}} Y_{k_{2}}\right]=0, \\
\mathrm{E}\left[X_{k_{1}} Y_{k_{2}}\right] & =-\mathrm{E}\left[X_{k_{2}} Y_{k_{1}}\right]=-\left(2 \pi \Delta f \Delta k \tau_{d}\right) \mathrm{E}\left[X_{k_{1}} X_{k_{2}}\right],
\end{aligned}
$$

where $\Delta k=\left|k_{1}-k_{2}\right|$. With these definitions we obtain the correlation function

$$
\rho_{f}(\Delta k \Delta f)=\mathrm{E}\left[H_{k 1} H_{k 2}^{*}\right]=\frac{1+j 2 \pi \tau_{d} \Delta f \Delta k}{1+\left(2 \pi \tau_{d} \Delta f \Delta k\right)^{2}} .
$$

Note that from (5) the marginal distribution of each channel gain $\left|H_{k}\right|^{2}$ follows an exponential distribution with $\mathrm{E}\left[\left|H_{k}\right|^{2}\right]=1, \operatorname{var}\left|H_{k}\right|^{2}=1$ and

$$
\operatorname{corr}\left(\left|H_{k}\right|^{2},\left|H_{k+\Delta k}\right|^{2}\right)=\frac{1}{1+\left(2 \pi \Delta f \Delta k \tau_{d}\right)^{2}}
$$

where $\operatorname{corr}(\cdot, \cdot)$ represents the correlation coefficient. In this paper, we consider a MIMO system with independent channel coefficients in the $N_{R} \times N_{T}$ channel matrix, $\boldsymbol{H}_{k}$, for all subcarriers $k$. This is a reasonable assumption in urban environments or when the antenna spacings and angle spreads at the transmitter and receiver are large. We consider correlations in frequency, but assume spatial independence. The non-zero eigenvalues of $\boldsymbol{H}_{k} \boldsymbol{H}_{k}^{\dagger}$ are denoted by $\lambda_{1}^{(k)}>\lambda_{2}^{(k)}>\cdots>\lambda_{m}^{(k)}$ where $m=\min \left(N_{R}, N_{T}\right)$, and the maximum eigenvalue is denoted by $\lambda_{\max }^{(k)}=\lambda_{1}^{(k)}$. 


\subsection{Adaptive Modulation System}

In this paper we use an adaptive modulation scheme in which the estimated subcarrier SNR values (via the maximum eigenvalues) are used to adjust the modulation scheme. We ignore any guard interval or cyclic prefix in the OFDM block. Further, we consider seven modulation options: outage, BPSK, QPSK, 8-PSK, 16-QAM, 32-QAM and 64-QAM. The adaptive modulation is implemented via a target BER. The highest order modulation is always selected such that the BER, computed from the maximum eigenvalue, remains below the target. Full details can be found in [15].

\section{ANALYSIS}

A closed form expression for the LCR of BER will be derived in this section. It is assumed that the entries of $\boldsymbol{H}_{k}$ are iid $\mathcal{C N}(0,1)$ with correlation properties in frequency and time defined by the Jakes process [15] and in (5) - (8). For this scenario, the LCR of the maximum eigenchannel was derived in [15]. Invoking the results in [15] and using (3), we derive an LCR formula for the BER process as below.

The LCR of BER for the $i$-th modulation scheme is denoted $\operatorname{LCR}_{i}\left(P_{T h}\right)$. This gives the LCR of the BER process across the threshold $P_{T h}$ assuming that modulation $i$ is used. The overall LCR of the BER process for adaptive modulation (AM) is given by

$$
\begin{aligned}
\operatorname{LCR}_{A M}\left(P_{T h}\right) & =L C R_{s}+L C R_{j} \\
& =\sum_{i=1}^{L} \operatorname{LCR}_{i}\left(P_{T h}\right)+\sum_{j=1}^{L} \operatorname{LCR}_{\lambda}\left(T_{j}\right),
\end{aligned}
$$

where $L$ is the number of alternative modulation modes. In (9), $L C R_{s}$ represents the LCR due to the smooth BER process and $L C R_{j}$ represents the LCR due to the jumps in BER which occur when the modulation changes. In (9), $L C R_{\lambda}\left(T_{j}\right)$ represents the LCR of $\lambda_{\max }^{(k)}$ across $T_{j}$ which results in a change of modulation and the summation is over all modulation changes which result in a crossing of BER across $P_{T h}$. Note that the overall $L C R_{A M}$ is given by a sum of the component LCRs since several threshold crossings of $\lambda_{\max }^{(k)}$ all result in a crossing of a single BER threshold.

The LCR behavior is shown schematically in Fig. 1. The smooth crossings occur when $\lambda_{\max }$ increases within a given modulation causing a downcrossing across $P_{T h}$. The jump crossings occur when $\lambda_{\max }$ decreases so that a higher order modulation can no longer be supported and the BER process jumps from one BER curve to another. If, during this jump, the BER downcrosses $P_{T h}$ then it contributes to the total LCR.

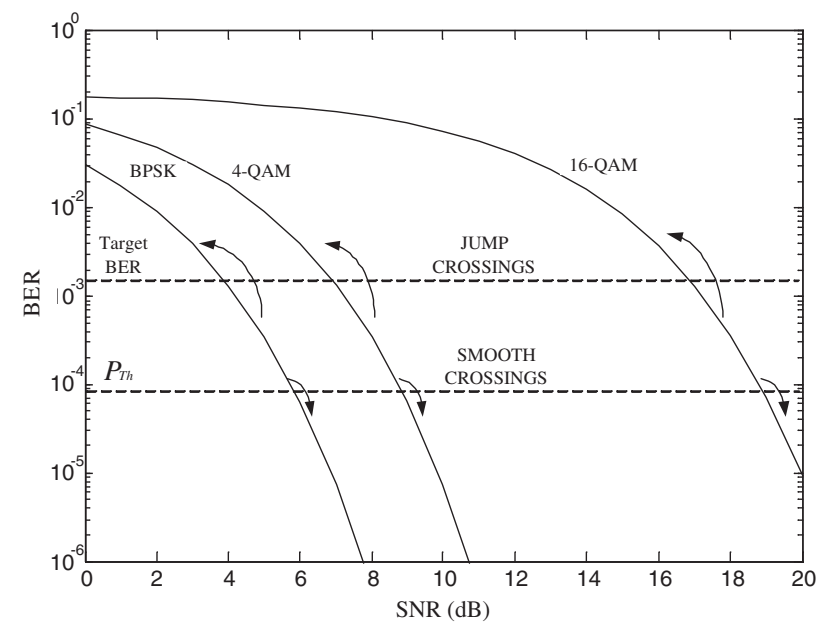

Figure 1: Schematic diagram of the LCR mechanism for AM.

Using the approximate expressions for the probability of bit error, $P_{E}$, given in [17] and using (3), $P_{E}$ can be written as

$$
P_{E} \approx c_{1} e^{-c_{2} \gamma_{\max }^{(k)}}
$$

where $c_{1}$ and $c_{2}$ are constants depending on the constellation being used. For MPSK, $c_{1}=\frac{1}{4}$ and $c_{2}=$ $8\left(2^{-1.94} \frac{\ln (M)}{\ln (2)}\right)$ and for MQAM, $c_{1}=\frac{1}{5}$ and $c_{2}=$ $\frac{3}{2(M-1)}$. Equation (10) is easily invertible, therefore the approximate expression for the SNR corresponding to a given BER threshold can written as

$$
\gamma_{\max }^{(k)} \approx-\frac{1}{c_{2}} \ln \left(\frac{P_{E}}{c_{1}}\right) .
$$

From [15], the closed form LCR approximation for the maximum eigenvalue of a MIMO OFDM system in the frequency domain is given by

$$
\operatorname{LCR}(T)=\sqrt{\frac{\pi}{r}} \frac{\tau_{d} \theta}{\Gamma(r)}(\theta \sqrt{T})^{r-0.5} e^{-\theta \sqrt{T}},
$$

where, $\Gamma(r)=\int_{0}^{\infty} t^{r-1} e^{-t} d t, r=\frac{\mathrm{E}[s]^{2}}{\operatorname{var}[s]}, \theta=\frac{\mathrm{E}[s]}{\operatorname{var}[s]}$, $T$ is the threshold value, $\tau_{d}$ is rms delay spread and $s=\sqrt{\lambda_{\max }^{(k)}}$. Note that these parameters depend solely on two moments of the maximum eigenvalue, and hence can be acquired from the distribution of the eigenvalues. More details on computing $\mathrm{E}[s]$ and $\operatorname{var}[s]$ can be found in [18]. 
In (12), replacing $T$ by $g\left(P_{T h}\right)$, the expression for $L C R_{i}\left(P_{T h}\right)$ can be written as

$\operatorname{LCR}_{i}\left(P_{T h}\right)=\sqrt{\frac{\pi}{r}} \frac{\tau_{d} \theta}{\Gamma(r)}\left(\theta \sqrt{g\left(P_{T h}\right)}\right)^{r-0.5} e^{-\theta \sqrt{g\left(P_{T h}\right)}}$.

The function $g($.$) is obtained by substituting (3) into$ (11),

$$
g\left(P_{T h}\right)=-\frac{\sigma^{2}}{E_{s} c_{2}} \ln \left(\frac{P_{T h}}{c_{1}}\right) .
$$

In the following section we verify our analytical results using Monte Carlo simulations.

\subsection{Analysis of non-maximal eigenchannels}

Note that all the derivations to date can be extended from MRT-MRC systems to MIMO-SVD systems. In MIMO-SVD, transmission down multiple eigenchannels is employed, possibly using all the channels. In this scenario, if $p \leq m$ eigenchannels are used, the transmit symbol $S_{k}$ is replaced by a $p \times 1$ transmit symbol vector and $\boldsymbol{b}_{k}$ is replaced by the $p$ eigenvectors corresponding to $\lambda_{1}^{(k)}>\lambda_{2}^{(k)}>\ldots>\lambda_{p}^{(k)}$. In terms of LCRs, the required changes are very minor. Equation (9) remains valid for each eigenchannel and is computed from (13)-(14). The only change is that $E(s)$ and $\operatorname{var}[s]$, which are required in (13), are now defined by $s=\sqrt{\lambda_{i}^{(k)}}$ for $i=1,2, \ldots, p$.

\section{SIMULATION RESULTS}

In this section, we compare the LCRs calculated using our formula with the results obtained from Monte Carlo simulations. The simulations were carried out for a $N=64$ subcarrier system and we used a subcarrier separation $\Delta f=0.3125 \mathrm{MHz}$. In all cases the product of $\Delta f$ and the rms delay spread, $\tau_{d}$, is set to 0.03125 which matches the HyperLan 2 standard. The results were plotted for the average SNR value per branch $\left(\frac{E_{s}}{\sigma^{2}}\right)$ equal to $2 d B$.

The accuracy of our formula for fixed modulation MIMO OFDM systems with different sizes is exhibited in Fig. 2. Note that the notation $\left(N_{R}, N_{T}\right)$ is used to denote the MIMO system size. A good match between the simulations and calculations is also shown for different constellation sizes in Fig. 3. Note that there are two sources of error in the LCR results. Firstly, the gamma process results in (13) are only approximations. Secondly, the calculated LCRs are in the continuous domain whereas the simulations are in the discrete domain. As a result, The LCR values calculated using (9) give higher values than the discrete version, since in the continuous domain there can be level crossings between the discrete points resulting in a higher value.

Figure. 2 shows LCR results for different MIMO system configurations. Here, we observe that for larger system sizes the maximum level crossings are occurring at lower BER thresholds. This is because with increasing system size the mean value of $\lambda_{\max }$ increases. Values of the means of $\lambda_{\max }$ for $(2,2),(2,4)$ and $(4,4)$ systems are 3.5, 6.19 and 9.77 respectively. With increasing system size, the effective SNR per branch increases and the BER decreases. Hence, the maximum level crossings occurs at lower BER thresholds. These results are very useful in comparing the fluctuations in BERs of various systems.

Figure. 3 shows LCR results for the BER with different constellation sizes in a $(2,2)$ MIMO system. Again, we can see a similar trend in the LCR curves. With a decrease in constellation size, the effective SNR per branch increases and the BER decreases. Hence, the maximum level crossings occur at lower BER thresholds.

The LCR results for a $(2,2)$ adaptive MIMO system using the maximum eigenchannel is given in Fig. 4. Note that the target BER is $10^{-2}$ so the BER axis does not go above $10^{-2}$. Considering Figs. $2-3$ we see that the region $\mathrm{BER} \leq 10^{-2}$ is where most of the $\mathrm{LCR}$ curves are decreasing. Hence the AM LCR curve in Fig. 4 follows a simple decreasing trend as BER drops. The AM causes jumps between modulations but since the LCR for each modulation is decreasing the overall LCR is also monotonic.

The LCR results for a $(4,4)$ adaptive MIMO system using the first three largest eigenchannels are given in Fig. 5. The LCR results are more complex here since the target BER is much higher at 0.2. In Fig. 5, the effect of modulation switching can be observed. At a given BER there is often a dominant modulation with large constellations at high BER and smaller constellations at low BER. Hence, as the BER drops, the AM LCR roughly follows the LCR curve of one modulation before switching to another. This explains the multiple peaks in the LCRs. Since the weaker eigenchannels cannot support low BERs very often, the LCR results for $\lambda_{3}$ are lower at low BER thresholds. Similarly since high BERs are more common for $\lambda_{3}$ the LCRs at high BER thresholds are larger for the $\lambda_{3}$ channel.

\section{CONCLUDING REMARKS}

Through the simulation results, we have verified that our formula provides very accurate approximations for the LCR of BER for an adaptive MIMO OFDM system. Our approximations can be used as a bench- 


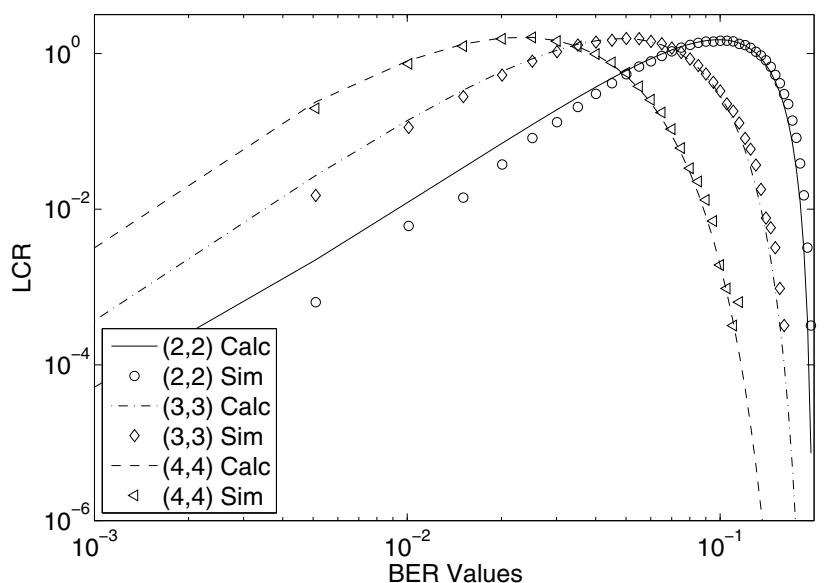

Figure 2: A comparison between simulated and calculated normalized LCRs of the BER for MIMO OFDM systems with different sizes (16-QAM, $N=64, \tau_{d}=$ 100ns).

mark for the system performance analysis. Although the results were presented only for the Jakes process, the analysis can be easily extended to arbitrary ACFs. Similarly, the approach can be extended to consider temporal variation. To the best of our knowledge, this is the first study of its kind, which considers BER variation over frequency and also level crossings with adaptive modulation.

\section{References}

[1] S. M. Alamouti, "A simple transmit diversity technique for wireless communications," IEEE J. Select. Areas Commun., vol. 16, pp. 1451-1458, Oct. 1998.

[2] S. Catreux, V. Erceg, D. Gesbert, and R. W. Heath, "Adaptive modulation and MIMO coding for broadband wireless data networks," IEEE Trans. Commun., vol. 40, no. 6, pp. 108-115, June 2002.

[3] J. C. Roh and B. D. Rao, "Adaptive modulation for multiple antenna channels," in Proc. 36th Asilomar Conf. on Signals, Systems, and Computers, vol. 1, Pacific Grove, CA, USA, 3-6 Nov. 2002, pp. 526-530.

[4] D. Gesbert, M. Shafi, D.-S. Shiu, P. Smith, and A. Naguib, "From theory to practice: An overview of MIMO time-space coded wireless systems," IEEE Trans. Commun., vol. 21, pp. 281-302, Apr. 2003.

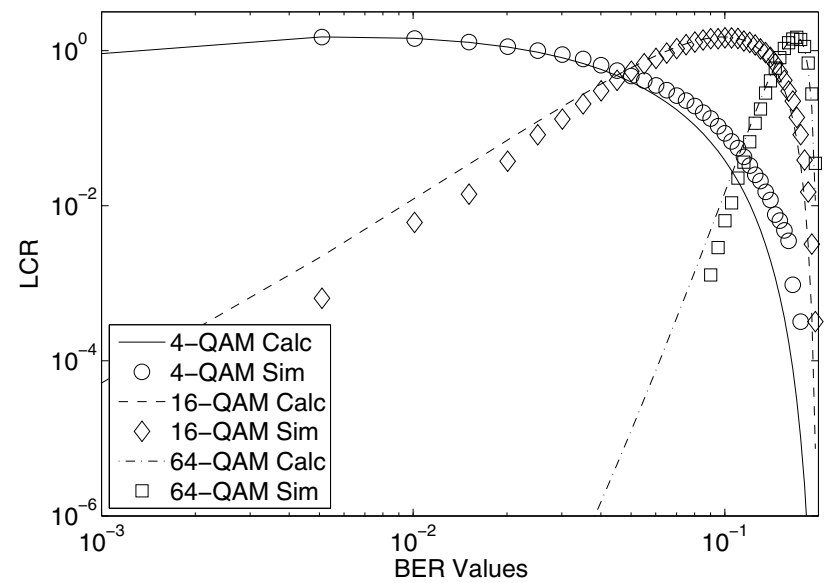

Figure 3: A comparison between simulated and calculated normalized LCRs of the BER in a MIMO OFDM system using different constellation sizes $\left(N_{R}=4\right.$, $\left.N_{T}=4, N=64, \tau_{d}=100 \mathrm{~ns}\right)$.

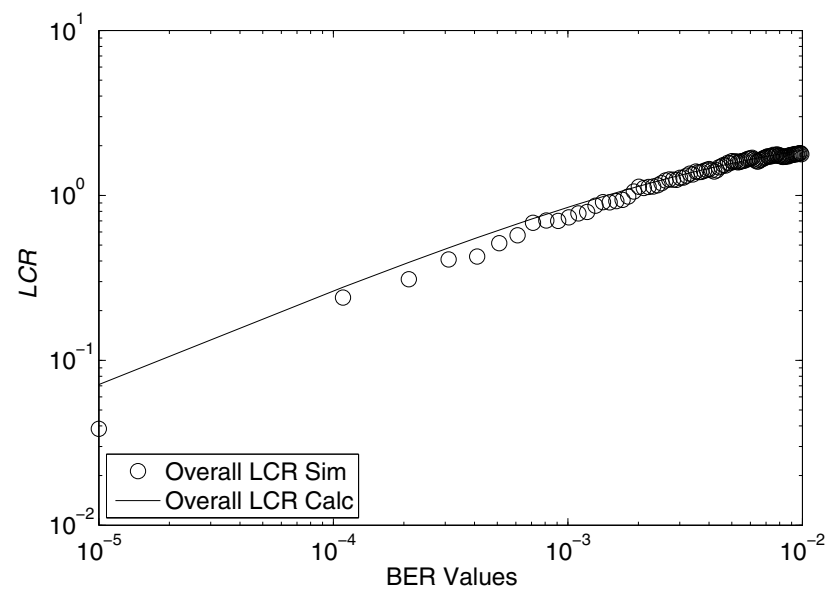

Figure 4: A comparison between the simulated and calculated normalized $L C R$ of the BER in an adaptive MIMO OFDM system $\left(N_{R}=2, N_{T}=2, N=64\right.$, $\left.\tau_{d}=100 \mathrm{~ns}\right)$ and target $\mathrm{BER}=10^{-2}$. 
[5] P. A. Dighe, R. K. Mallik, and S. S. Jamuar, "Analysis of transmit-receive diversity in Rayleigh fading," IEEE Trans. Commun., vol. 51, pp. 694703, Apr. 2003.

[6] A. Zanella, M. Chiani, and M. Z. Win, "Performance of MIMO MRC in correlated Rayleigh fading environments," in Proc. IEEE Vehicular Technology Conf., vol. 3, Stockholm, Sweden, 30 May-1 June 2005, pp. 1633-1637.

[7] A. Maaref and S. Aissa, "Closed-form expressions for the outage and ergodic Shannon capacity of MIMO MRC systems," IEEE Trans. Commun., vol. 53, pp. 1092-1095, July 2005.

[8] M. Kang and M. S. Alouini, "Largest eigenvalue of complex Wishart matrices and performance analysis of MIMO MRC systems," IEEE J. Select. Areas Commun., vol. 21, pp. 418-426, Apr. 2003.

[9] K. K. Mukkavilli, A. Sabharwal, E. Erkip, and B. Aazhang, "On beamforming with finite rate feedback in multiple antenna systems," IEEE Trans. Inform. Theory, vol. 49, pp. 2562-2579, Oct. 2003.

[10] P.-H. Kuo, P. J. Smith, and L. M. Garth, "Level crossing analysis of MIMO eigenmodes and associated channel metrics," in preparation.

[11] A. Giorgetti, M. Chiani, M. Shafi, and P. J. Smith, "Level crossing rates and MIMO capacity fades: Impacts of spatial/temporal channel correlation," in Proc. IEEE ICC, Anchorage, AK, USA, May 11-15, 2003, pp. 3046-3050.

[12] A. Giorgetti, P. J. Smith, M. Shafi, and M. Chiani, "MIMO capacity, level crossing rates and fades: The impact of spatial/temporal correlation," $J$. Communications and Networks, vol. 5, pp. 104115, June 2003.

[13] A. Abdi, C. Gao, and A. M. Haimovich, "Level crossing rate and average fade duration in MIMO mobile fading channels," in Proc. IEEE VTC Fall, Orlando, FL, USA, Oct 5-9, 2003, pp. 3164-3168.

[14] P. Ivanis, D. Drajic, and B. Vucetic, "Level crossing rates in transmit beamforming systems," IEEE Commun. Lett., vol. 11, pp. 246-248, Mar. 2007.

[15] K. P. Kongara, P.-H. Kuo, P. J. Smith, L. M. Garth, and A. Clark, "Performance analysis of adaptive MIMO OFDM beamforming systems," in Proc. IEEE Int'l. Conf. on Communications, Beijing, China, 19-23 May 2008, pp. 4359 - 4365.

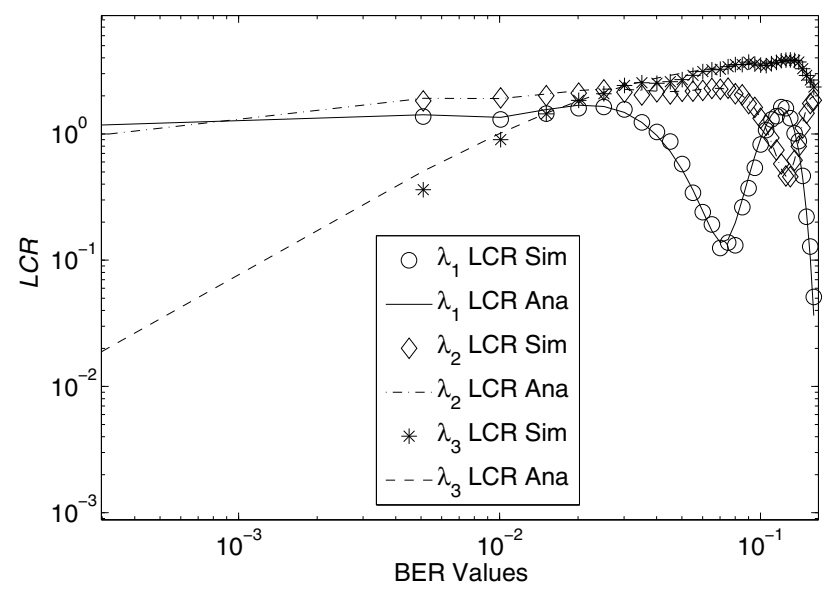

Figure 5: A comparison between the simulated and calculated normalized $L C R$ of the BER in an adaptive MIMO OFDM system when the first three eigenchannels $\left(\lambda_{1}, \lambda_{2}, \lambda_{3}\right)$ are used $\left(N_{R}=4, N_{T}=4, N=64\right.$, $\left.\tau_{d}=100 \mathrm{~ns}\right)$.

[16] W. C. Jakes, Ed., Microwave Mobile Communications. New York, NY, USA: IEEE Press, 1994.

[17] S. T. Chung and A. J. Goldsmith, "Degrees of freedom in adaptive modulation: a unified view," IEEE Trans. Commun., vol. 49, pp. 1561-1571, Sept. 2001.

[18] A. Zanella, M. Chiani, and M. Z. Win, "On the marginal eigenvalues distribution of Wishart matrices," University of Bologna, Bologna, Italy, IEIIT B0-07-06 Tech. Rep., Dec. 2006. 